\title{
The Problem of Ether Drift*
}

\section{By Dr. C. V. Drysdale, c.b., o.b.e.}

\section{Analysis of The Michelson-Morley OBSERVATIONS}

$\mathrm{I}^{\mathrm{T}}$ should be noted that as the displacement of the fringes is proportional to the square of the velocity of drift, a velocity of $10 \mathrm{~km}$. $/ \mathrm{sec}$. only corresponds to a displacement of about $0 \cdot 1$ of a fringe, and, as the width of the fringes could not be kept quite constant, visual estimation to the nearest $0 \cdot 1$ fringe was all that was possible for the enormous number of observations which have been made. Hence the existence of a drift could only be determined by averaging a large number of sets of observations at sixteen equally-spaced azimuths, and by employing a Henrici harmonic analyser to determine the component of double period in a revolution. The results of this analysis reveal a small full-period component which was accounted for satisfactorily by Hicks in 1902 as due to the small inclination of the mirrors required to produce the fringes, and a relatively large double-period component, the amplitude of which represents the component of the drift in the plane of the interferometer. The sum of the higher harmonics is usually relatively small, which indicates that the drift effect is real. This procedure seems first to have been adopted for the Mount Wilson observations of 1921, which explains why various interpretations could be put upon the earlier Michelson-Morley results. Miller has, however, since applied it to these earlier observations, and has therefore come to the conclusions above stated.

But the harmonic analyser gives the phase as well as the amplitude of the double period component, and therefore the azimuth of the horizontal component of the drift. By taking observations at several intervals during the day and correlating the magnitudes and azimuths of the drift with the geographical position of the interferometer and the movements of the earth, it became clear that the drift observed was not chiefly caused by the orbital motion but by a motion of the whole solar system in a direction nearly perpendicular to the plane of the orbit. Further observations were therefore made at Mount Wilson in April, August and September 1925, and in February 1926, in order if possible to determine the sense as well as the direction of the absolute motion, as the interferometer is of course 'bi-directional'. The four apices of the motion, which can be determined independently from the amplitudes and the phases of the observed drifts, were found to lie almost exactly on a circle which is the 'aberration orbit'

\footnotetext{
* Continued from p. 798.
}

of the earth ; and this led to the conclusion that the solar system must be moving towards the constellation Dorando in a direction $7^{\circ}$ from the south pole of the ecliptic, and with a velocity of about $208 \mathrm{~km} . / \mathrm{sec}$.

The above velocity was derived by calculation from the orbital velocity of the earth and the directions of the drift at the various epochs; and as it is about twenty times the velocity of the drift given by the interferometer, it would appear either that the earth imparts a material fraction of its velocity to the ether near its surface, which is highly improbable, or that the Fitzgerald-Lorentz contraction differs by five per cent from the truth.

It is impossible to read Prof. Miller's account of these laborious researches, with the meticulous care they reveal, and the remarkable consistency of the results, without feeling that a drift has actually been observed; but, on the other hand, Michelson himself, Piccard and Stahel, Kennedy, and Zoos have recently made careful trials with various modifications of the interferometer, and have all failed to detect any definite evidence of drift. Miller, however, is definitely of the opinion that the drift can be screened by thick walls or metallic enclosures, and points out that nearly all these instruments were so enclosed. At a conference held at Mount Wilson Observatory in 1927, at which Michelson, Miller, Lorentz and Kennedy were present, Miller was the only champion of a positive result, and Lorentz confessed that at a previous conference at Düsseldorf in 1898 the conviction against the detection of at least a first order effect became so strong that attention was only paid to those papers which announoed negative results. Nevertheless, the impression created by Miller was considerable, and Michelson proposed to carry out a further trial with an invarframed interferometer and further optical refinements.

So the matter rests at the present time, but it is clear that it is in a very unsatisfactory state, and that it cannot be settled by any improvements in the Michelson-Morley apparatus, or by any other method based on a second order effect, on account of the doubt concerning the exact amount of the contraction or rather distortion which theory indicates must exist, apart from the extreme delicacy of the method.

\section{Is a First-Order Effect Unobtainable ?}

It is evident, therefore, that a first-order effect is imperatively needed to clear up the problem, but the complete failure of all attempts to detect 
a change of deviation or of the time of transit through dense transparent media, combined with the theoretical impossibility of doing so if the Fresnel drag formula is accurately true, has led to a universal disbelief in the possibility of observing a first-order effect; just as the supposed null results of all experiments by the Michelson-Morley method, combined with the Fitzgerald-Lorentz contraction formula, have evoked disbelief in the possibility of detecting a second-order effect. Indeed, the denial of any possibility of detecting motion through the ether has become a cardinal assumption of the general theory of relativity. This denial has been emphatically stated by Prof. R. W. Wood in the last edition of his "Physical Optics", where he says that the first postulate of the theory of relativity "amounts to saying that motion through the ether (if the ether exists at all) will be wholly without influence upon all optical experiments made with terrestrial sources of light".

But the Maxwellian theory, as developed by Drude and by Lorentz, definitely indicates the possibility of observing a first-order effect, and as a matter of fact such an effect was actually observed, as early as 1859, by Fizeau, who conceived it from the then existing theory. As is well known, Drude was the first to introduce the modern conception of mutually attracting ions into optical theory, and thereby to develop a remarkably successful theory of dispersion as well as of most other optical phenomena. Following on the same lines, Lorentz in 1895 published an important paper on electrical and optical phenomena in moving bodies, in which he established the Fresnel drag formula on a sound theoretical basis and gave an outline of the theory of the "contraction" effect. These important developments have helped greatly to clarify our conceptions.

One fundamentally important conclusion which emerges from the above theories and from the moving water experiments of Fizeau, Michelson and Morley, and Zeeman, is that motion through the ether has a definite meaning and measurable effect. The failure of all interferometer and deviation experiments with dense media to reveal any effect of the earth's motion through the ether is simply due to the fact that the effects which are undoubtedly produced when the light enters the dense medium are exactly cancelled by its subsequent passage through the medium. In the case of a block of dense medium interposed in an interferometer and moving in the direction of propagation, the increase in the velocity of propagation due to the motion is exactly balanced by the increase of the effective length of the block. A transverse motion, on the other hand, produces a change in the angle of incidence of the wave front equal to the aberration, and therefore a corresponding deviation of the refracted wave front, but this deviation is exactly neutralised by the transverse drag of the medium as the light proceeds. The fact that no resultant deviation can be observed is therefore an experimental proof of a deviation of the refracted wave front, unless we assume that the ether near the surface of the earth is carried along with it, which would be contrary both to the Maxwell-Lorentz theory and to the observations of astronomical aberration.

Both theory and experiment therefore agree in indicating that a transverse drift alters the refraction at the surface of a dense medium, so that it is clear that any effect of that change at the surface which is unmodified by subsequent passage of the light through the medium should be capable of observation.

Such an effect is available in the polarisation which occurs at oblique incidence on an isotropic dense medium. According to the Maxwellian theory, this polarisation depends simply upon the inclinations of the incident and refracted wave fronts to the surface; and the angle of complete or maximum polarisation of the reflected light is given by $\tan \theta=n$. Jamin has shown that this polarisation is practically complete at the polarising angle for substances having a refractive index of about $1 \cdot 46$, so that it would appear that a simple blackened-glass polariscope should show a change of about 37 seconds in its polarising angle when it is rotated in the plane of the earth's orbit.

of course, such a small effect would probably be difficult to measure, but it may be amplified considerably by making use of the rotatory effect of an oblique plate on an already polarised beam; and this was the method employed by Fizeau, who used a pile of forty thin glass plates of slightly prismatic form so as to deviate the reflected beams away from the transmitted beam, the rotation of which was observed. His observations, which were conducted with three different dispositions of the apparatus, gave rotations in some cases of as much as $2 \frac{1}{2}$ degrees; and they varied with the orientation of the apparatus and time of day as anticipated. They also agreed fairly closely with his predictions from the then existing theory, but these are perhaps open to criticism.

There can be little doubt, however, in the light of our present knowledge, that the basis of Fizeau's method is sound ; and it is a remarkable testimony to his prescience and ingenuity that he should apparently have realised the necessity for what may be called a "surface method" and have devised such an ingenious method of carrying it into effect at such an early date. Unfortunately, he does not seem to have realised its importance in relation to the ether drift problem, and devised it primarily for the purpose of extending his verification of the 
Fresnel drag formula to solid substances; neither

did he give any cogent reasons for having adopted this particular method; so that his remarkable research has scarcely attracted any attention.

After a comprehensive survey of the problem, however, the present writer is convinced that the surface polarisation method does provide a means of measuring a first-order effect of ether drift, and that Fizeau's experiments have actually demonstrated such an effect. There seem to be various possibilities for simplifying the method, with which it is hoped to experiment before long; but in the meantime, this account of Miller's and of Fizeau's experiments may do something to revive interest in this important problem and to dispel the prevalent belief that it is insoluble.

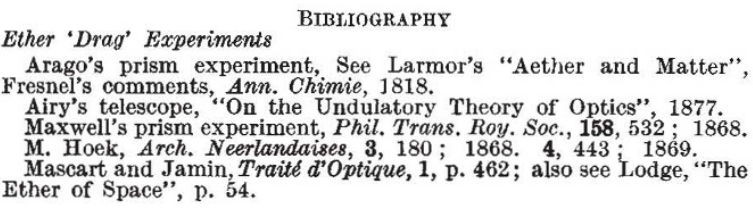

Velocity of Light in Moving Water

Fizeau, Ann. Chimie, 57, 385; 1859.

Michelson and Morley, Amer. J. Sci., 31, 377 ; 1886.

P. Zeeman, K. Akad. Wetenschappen, 17, 445 ; 18, 398; 1914.

Michelson-Morley Experiments

A. A. Michelson, Amer. J. Sci., 22, 120; 1881. 23, 395; 1882 Phil. Mag. (5), 13, 236; 1882.

A. A. Michelson and E. W. Morley, Amer. J. Sci. (3), 34, 333 1887. Phil. Mag. (5), 24, 449 ; 1887 .

F. W. Morley and D. C. Miller, Phil. Mag. (6), 9, 680 ; 1905.

D. C. Miller, Proc. Nat. Acad. Sci., 11, 306; 1925. "The Science of

Musical Sounds"' 123 ; 1916. J. Frank. Inst., 181, 51; 182, 285 ; R. J. Kennedy, Proc. Nat. Acad. Sci., 12, 621; 1926. Astroph. J. 68, $367 ; 1928$.

A. Piccard and E. Stahel, C.R., 183, $420 ; 1926.185,1198 ; 1927$. A. A. Michelson, F. G. Pease and F. Pearson, NAture, 123, 88 ; 1929. J. Opt. Soc. Amer., 18, 181; 1929.

G. Joos, Phys. Rev., 45, 114; 1934. NATURE, 128, 729; 1931.

Theory

W. M. Hicks, Phil. Mag. (6), 3, 9, 256 and 555; 1902. NATURE, 65, $343 ; 1902$.

L. Silberstein, Phil. Mag. (6), 39, 161; 1920.

Fitzgerald, see O. J. Lodge, "Past Years", p. 204 ; 1902.

H. A. Lorentz, "Versuch einer Theorie der elektrischen und optischen Erscheinungen in bewegten Körpern, Leyden" ; 1895.

P. Drude, "Theory of Optics", chap. viii.

O. J. Lodge, "Aberration Problems", Phil. Trans., 184, 727 ; 1893 O.: J. Lod

Fizeau's Polarization Experiment

H. Fizeau, Ann. de Chimie, 58, $129 ; 1860$.

\section{The Study of Behaviour*}

\section{By Dr. E. S. Russell, O.B.E.}

$I^{\mathrm{T}}$ was Descartes who imposed upon European thought for at least two centuries, and upon biology for much longer, that 'bifurcation' of Nature into matter and mind which has raised so many insoluble problems for philosophy, and diverted biology from its true method. As to its effect on philosophy, Prof. A. N. Whitehead writes :

"T.he seventeenth century had finally produced a scheme of scientific thought framed by mathematicians, for the use of mathematicians. ... The enormous success of the scientific abstractions, yielding on the one hand matter with its simple location in space and time, on the other hand mind, perceiving, suffering, reasoning, but not interfering, has foisted on to philosophy the task of accepting them as the most concrete rendering of fact. Thereby, modern philosophy has been ruined. There are the dualists, who accept matter and mind as on equal basis, and the two varieties of monists, those who put mind inside matter, and those who put matter inside mind. But this juggling with abstractions can never overcome the inherent confusion introduced by the ascription of misplaced concreteness to the scientific scheme of the seventeenth century."

Actually, instead of being the most concrete of realities, both matter and mind are highly abstract concepts, the product of the reflective intelligence working upon the data of immediate experience.

* From the presidential address before Section D (Zoology) of the British Association, delivered at Aberdeen on September 6.
There is given in individual experience only the perceiving subject and his objective world. This dualism does not correspond, is not synonymous with, the dualism of matter and mind. Subjective experience as we know it directly is a function of organism, not of pure mind ; objective experience is a relation between organism and other processes or events. The concept of matter is arrived at by abstracting from the data of sense, by leaving out the 'secondary qualities' such as colour, smell and sound, and retaining the so-called 'primary qualities' of resistance and extension, with location in time and space.

By accepting this abstract definition or concept of matter, we substitute for the objective world of perception a symbolic or conceptual world of discrete material particles, which we may call the 'world of matter'. This world of matter the materialist takes to be in some sense more real than the perceptual and colourful world from which he has derived it. Actually it is less real, less concrete. It is important to remember that the world which we perceive through the senses, with its shapes, colours, smells, tastes and so on, is not identical with the conceptual 'world of matter' ; we do not perceive 'matter' at all, any more than we perceive mind; we perceive things or relations or events.

Complementary to this abstract material universe is the concept of mind as an inextended, immaterial, thinking entity, and this also is 